Article

\title{
Development of Rapid Extra Virgin Olive Oil Quality Assessment Procedures Based on Spectroscopic Techniques
}

\author{
Paola Baltazar ${ }^{\circledR}$, Natalia Hernández-Sánchez *®i , Belén Diezma ${ }^{\circledR}$ and Lourdes Lleó \\ Laboratory of Physical Properties-Advanced Technologies in Agrifood (LPF_Tagralia), ETSIAAB, Universidad \\ Politécnica de Madrid, Av. Puerta de Hierro, 2-4, 28040 Madrid, Spain; \\ paola.baltazar.delcastillo@alumnos.upm.es (P.B.); belen.diezma@upm.es (B.D.); lourdes.lleo@upm.es (L.L.) \\ * Correspondence: n.hernandez@upm.es
}

Received: 25 November 2019; Accepted: 23 December 2019; Published: 26 December 2019

\begin{abstract}
The main objective of this study was to evaluate the feasibility of developing multivariate models to estimate physico-chemical characteristics and antioxidant content of extra virgin olive oil from fluorescence spectra obtained at specific excitation wavelengths. Six replicates of each extra virgin olive oil sample were contained in clear glass bottles. Two replicates were subjected to four weeks of natural indirect light; two bottles for two days; and the third couple were kept it in darkness as a control. For each pair, one bottle was used for spectroscopic measurements and the other was sent to an accredited external laboratory to obtain physico-chemical measurements: acidity, peroxide index, $K_{270}, K_{232}$, total tocopherols, $\alpha$-tocopherol, $\beta$-tocopherol and $\gamma$-tocopherol. Fluorescence emission spectra were acquired at different excitation wavelengths: $326 \mathrm{~nm}, 350 \mathrm{~nm}$ and $365 \mathrm{~nm}$ and partial least squares regression (PLSR) models were developed. The highest $R^{2}$ values were found for excitation at $350 \mathrm{~nm}$, reaching almost 0.9 in most of the parameters.
\end{abstract}

Keywords: tocopherol; EVOO; fluorescence; olive; physico-chemical

\section{Introduction}

The production and consumption of olive oil has increased and extended from the Mediterranean area to other regions of the world. The growing interest in this product, especially in extra virgin olive oil (EVOO), is based on its beneficial properties for health and on its sensory attributes, since it is rich in monounsaturated fatty acids and antioxidant compounds such as polyphenols and tocopherols.

In this context, EVOO marketing is more demanding in terms of quality assurance. The olive oil sector is subject to compliance with regulations that affect its commercialization [1-3]. Regulations establish the categorization of the olive oil according to numerous physico-chemical characteristics that are analyzed in accredited laboratories, as well as to organoleptic evaluation by accredited tasting panels. These analyses are usually laborious, slow and expensive.

Regulation (EEC) No 2568/91 (amended by Regulation (EU) No 1348/2013) establishes a decision tree for verifying whether an olive oil sample is consistent with the category declared. The decision tree requires the hierarchical determination of quality parameters such as acidity, peroxide value, extinction coefficients at $270 \mathrm{~nm}$ and $232 \mathrm{~nm}\left(\mathrm{~K}_{270}, \mathrm{~K}_{232}\right)$, and $\Delta \mathrm{K}$. The respective limit values for EVOO are shown in Table 1. Such determinations include titration and spectrophotometric analysis in the ultraviolet range, which involves the use of solvents and reagents. 
Table 1. Limit values for the physico-chemical characteristics for EVOO category in Regulation (EU) No 1348/2013 that amends Regulation (EEC) No 2568/9.

\begin{tabular}{ccccc}
\hline Acidity (\%) & $\begin{array}{c}\text { Peroxide Value } \\
\mathbf{m E q ~ O}_{\mathbf{2}} / \mathbf{k g}\end{array}$ & $\begin{array}{c}\text { UV Spectrometry } \\
\mathbf{K}_{\mathbf{2 7 0}}\end{array}$ & $\begin{array}{c}\text { UV Spectrometry } \\
\mathbf{\Delta K}\end{array}$ & $\begin{array}{c}\text { UV Spectrometry } \\
\mathbf{K}_{\mathbf{2 3 2}}\end{array}$ \\
\hline$\leq 0.8$ & $\leq 20$ & $\leq 0.22$ & $\leq 0.01$ & $\leq 2.50$ \\
\hline
\end{tabular}

Fluorescence spectroscopy has been successfully used for analysis of EVOO quality [4,5], and is less time-consuming and more cost-efficient compared to other analytical procedures. The high sensitivity of fluorescence spectroscopy allows detection of the presence of the fluorescent compounds.

The fluorescent compounds contained in olive oil are directly associated with its beneficial effects on health, as well as oxidation resistance and freshness level. Such compounds are polyphenols and tocopherols, primary and secondary oxidation products, chlorophylls and pheophytins [6]. Moreover, these compounds are related to the main quality criteria established in regulations. Consequently, procedures for estimating physico-chemical characteristics could be developed based on fluorescence spectroscopic data, as proposed in this work.

Primary oxidation products are related to the regulated parameters peroxide index and $\mathrm{K}_{232}$. These products are not detectable by panels. Early detection of oxidation processes is of paramount importance since it identifies the samples with potential secondary oxidation problems.

Secondary oxidation products (including a variety of low molecular weight aldehydes, ketones, alcohols and short hydrocarbon chains) are related to the regulated parameter $\mathrm{K}_{270}$.

Chlorophyll and its derivatives, not included in the regulations, act as antioxidants under darkness conditions, and as pro-oxidant under light conditions. These compounds are markers of photo-oxidation processes. In addition, oxidation products and chlorophylls are useful for freshness evaluation and fraud detection [5].

The major interest in polyphenols and tocopherols arises from their antioxidant activity.

Previous studies on fluorescence spectra of olive oil [5-12] revealed the characteristic spectral patterns of different oil qualities according to the presence of peaks at certain wavelengths and to their signal intensity. Such patterns allowed the development of multivariate models for discrimination between categories [9-11].

Additionally, spectral patterns can be useful for the evaluation of olive oil quality by estimating the physico-chemical characteristics related to fluorescence compounds, which are excited by specific wavelength ranges. Therefore, selection of the optimal wavelengths of excitation is of paramount importance.

During the last decade great effort has been made to develop environmentally friendly methodologies for assessing the authentication of EVOO [5]. Most of the works that applied fluorescence spectroscopy focused on the assessment of adulterated EVOO (detection and quantification of adulteration). Controlled addition of adulterants consisting of the mix of EVOO with cheaper oils (refined olive oils, pomace olive oils, seed oils, etc.) was studied.

EVOO samples were mixed with increasing quantities of sunflower oil and the excitation wavelength was $350 \mathrm{~nm}$. The emission spectra were acquired from 350-700 nm [12]. Such spectra presented similar patterns as those showed by previous works [10,11]. Chlorophyll produced a dominant peak at $670 \mathrm{~nm}$ that decreased with the adulteration level. A broad region of fluorescence with local peaks from $400-600 \mathrm{~nm}$ was attributed to oxidation products, tocopherol and carotenoids. High accuracy of the models was obtained when fluorescence spectra were submitted to PCA and PLSR for detection of different concentrations of added sunflower oil.

A methodology based on a neural network (supervised multilayer perceptrons) was developed for the detection of adulteration of EVOO with increasing quantities of expired EVOO, and for the identification of storage conditions (light exposition, type of bottle) [13]. Results showed high classification accuracy (up to $90 \%$ ) when the source of excitation light was UV LED or laser diode. 
The main differences of spectral patterns were derived from the chlorophyll dominant peak, and weak response was found within the range from $400-600 \mathrm{~nm}$.

Synchronous fluorescence measurements were carried out with diluted samples in hexane and different classification methods were developed for discriminating EVOO, expired EVOO, refined, and expired refined olive oil [14]. Similar trends in the spectra to those in [13] were found. A band observed in the range of 270-300 nm was ascribed to the emission of tocopherols and tocotrienols. The characteristic peak of the chlorophylls and pheophytins was also obtained from 660-700 nm. The study has shown that non-expired extra virgin, non-expired refined, expired extra virgin, and expired refined olive oil samples exhibit significant differences in their synchronous fluorescence spectral patterns. The tocopherol and chlorophyll band intensity decreases, and simultaneously, a new emission band appears in the 320-380 $\mathrm{nm}$ wavelength range.

Mixes of EVOO with lower quality olive oils were evaluated by [15]. The fluorescence emission signal of vitamin E (tocopherol) appeared within the range from 310-380 nm, with excitation at $290 \mathrm{~nm}$; and the signal from chlorophylls with excitation at $405 \mathrm{~nm}$. Spectral patterns from mixtures were different from EVOO as composition was significantly altered.

Although the results of these recent works highlighted that fluorescence could be used for quantifying specific constituents, few researches have been conducted to estimate quantitatively oil compounds or physico-chemical characteristics. Successful results were found for some physico-chemical characteristics of a set of samples with a wide range of degradation produced by heat treatments [8].

However, characterization of olive oils that present subtle degradation or early identification of first oxidation stages is still a challenge that the present work aimed to accomplish. The main objective was to evaluate the feasibility of developing multivariate models to estimate compounds and physico-chemical characteristics of extra virgin olive oils showing very little degradation. The selection of the most useful excitation wavelength was a major issue for achieving such purpose.

The new procedure would improve the strategic management plans of EVOO producers as it would immediately provide them with valuable information on the quality of the oil they are producing, which would show a minimum degradation. Other links of the commercial olive oil chain could benefit from such technique.

\section{Materials and Methods}

\subsection{Initial Samples}

Eight samples of EVOO were provided by Finca La Pontezuela S.L. oil mill (Designation of Origin Montes de Toledo, Toledo, Spain). Five samples corresponded to the emblematic variety of the Designation of Origen Cornicabra, growing under different agronomical conditions (orchard hedgerows orientation, irrigation, fertilization, etc.). In addition, one sample of each variety: Picual, Hojiblanca and Redondilla was provided (Table 2).

Table 2. Codification of the initial samples.

\begin{tabular}{cccc}
\hline Num. & Variety & Abbreviation & Sample \\
\hline 1 & Cornicabra & C & C-D5 \\
2 & Picual & P & P-D7 \\
3 & Cornicabra & C & C-D8 \\
4 & Hojiblanca & H & H-D10 \\
5 & Redondilla & R & R-D11 \\
6 & Cornicabra & C & C-D16 \\
7 & Cornicabra & C & C-D17 \\
8 & Cornicabra & C & C-D19 \\
\hline
\end{tabular}




\subsection{Initial Samples Treatments}

Six aliquots were obtained from each initial sample, and contained in clear glass bottles, $100 \mathrm{~mL}$ volume. As illustrated in Figure 1, paired aliquots were exposed to natural illumination for three different periods in order to generate a final set with high variability in their oxidation state. Bottles were placed on a table at $1 \mathrm{~m}$ from a window. The samples received natural indirect sunlight along the period of exposition.

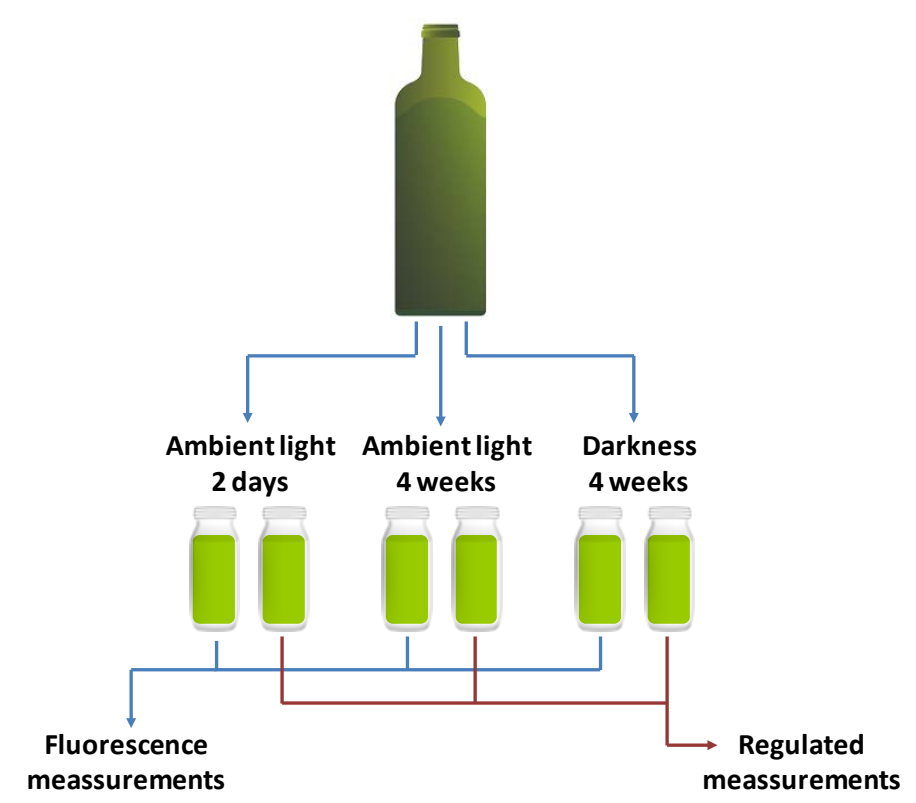

Figure 1. Illustration of light exposure treatments and measurements carried out on each replicate.

Two out of six aliquots from each initial sample were exposed to natural illumination for two days, two were exposed for four weeks, and two aliquots were maintained under darkness conditions. One bottle of each pair was used for fluorescence measurements. The other bottle was sent to an officially accredited laboratory to obtain physico-chemical characteristics included in Regulation (EEC) No 2568/9 and content of antioxidant compounds (Figure 1). The experimental design provided a total of 24 paired final samples ( 8 initial samples $\times 3$ illumination treatments $=24$ samples) characterized by fluorescence spectroscopic data and analytical data.

\subsection{Selection of the Excitation Wavelengths with 3D Fluorescence Spectroscopy}

Three-dimensional front-face fluorescence spectra were obtained from a set of seven EVOO samples including Arbequina, Picual, Hojiblanca and Cornicabra varieties. A spectrofluorometer (LS45, Perkin-Elmer, Wellesley, MA, USA) equipped with a Xenon lamp source, excitation and emission monochromators and a front-face sample-cell holder was used. The excitation wavelengths ranged from 230-646 nm (at $4 \mathrm{~nm}$ increments) and the emission wavelengths ranged from 250-698.5 nm (spectral resolution $0.5 \mathrm{~nm}$ ). Slit widths of excitation and emission monochromators were set at $10 \mathrm{~nm}$. Quartz cuvettes $(10 \mathrm{~mm} \times 10 \mathrm{~mm} \times 45 \mathrm{~mm})$ were filled with the oil samples without prior preparation.

For each excitation wavelength the sum of the signal intensities along the emission wavelengths from 250-698.5 $\mathrm{nm}$ was computed. Such excitation spectra revealed the wavelengths, appearing as local maxima, that gave rise to emission signal from fluorescent oil compounds and, therefore, potentially provided relevant information on the oil quality.

These local maxima were considered for the selection of the most suitable excitation wavelengths to study the feasibility of developing estimation models for oil quality parameters. 


\subsection{Fluorescence Spectroscopy}

The fluorescence spectra were obtained using the QE Pro spectrometer (Ocean Optics, Inc., Dunedin, FL, USA) with a CCD image sensor and detection wavelengths ranging from $197 \mathrm{~nm}$ to $998,763 \mathrm{~nm}$. The intensity of the light emitted by the fluorescent compounds in olive oil is weak. Thus, a slit of $200 \mu \mathrm{m}$ size was used in order to receive as much light as possible without compromising resolution. Integration time was set to $10,000 \mathrm{~ms}$ and 3 repetitions per measurement were averaged.

Measurements were carried out in a right-angle setup using quartz cuvettes $(10 \mathrm{~mm} \times 10 \mathrm{~mm} \times 45 \mathrm{~mm})$ filled with the oil samples without prior preparation.

The high-power Xenon light source HPX-2000 (Ocean Optics, Inc., Dunedin, FL, USA), with a compartment to accommodate optical filters, was used to excite the samples. Three different bandpass filters (OptoSigma ${ }^{\circledR}$, Santa Ana, CA, USA) centred at $326 \mathrm{~nm}, 350 \mathrm{~nm}$ and $365 \mathrm{~nm}$ (10 nm bandwidth) were selected for the acquisition of fluorescence spectra (Table 3). The maximum transmittance was about $30 \%$ for the three filters. In the end, 72 spectra were acquired $(24$ oil samples $\times 3$ filters $=$ 72 spectra).

Table 3. Characteristics of the bandpass filters used to select the excitation wavelength.

\begin{tabular}{ccc}
\hline Model & Central Wavelength & Bandwidth \\
\hline VPF-25C-10-25-32600 & $326 \mathrm{~nm}$ & $10 \mathrm{~nm}$ \\
VPF-25C-10-25-35000 & $350 \mathrm{~nm}$ & $10 \mathrm{~nm}$ \\
VPF-25C-10-25-36500 & $365 \mathrm{~nm}$ & $10 \mathrm{~nm}$ \\
\hline
\end{tabular}

Fluorescence spectra were denoised by applying the Savitsky-Golay smoothing algorithm with a window of 5 data, adjusted to a polynomial of degree 2, without application of differentiation. Further analysis was performed on the spectral region corresponding to the emission of the oxidation products and the tocopherols according to [7,8]. For excitation around $326 \mathrm{~nm}$ (BW $10 \mathrm{~nm}$ ), the emission wavelength range varied from $340 \mathrm{~nm}$ to $550 \mathrm{~nm}$. For excitation around $350 \mathrm{~nm}(\mathrm{BW} 10 \mathrm{~nm})$ the emission range varied from $365 \mathrm{~nm}$ to $550 \mathrm{~nm}$. For excitation around $365 \mathrm{~nm}$ (BW $10 \mathrm{~nm}$ ) the emission range varied from $380 \mathrm{~nm}$ to $550 \mathrm{~nm}$.

\subsection{Physico-Chemical Determinations}

An officially accredited laboratory carried out the determination of the oil characteristics summarized in Table 4, which included physic-chemical characteristics established in Regulation (EU) No 1348/2013 and the content of antioxidant compounds such as tocopherols.

Table 4. Determinations carried out by the accredited external laboratory.

\begin{tabular}{cc}
\hline Test & Units \\
\hline Acidity & $\%$ ac. Oleic \\
Peroxide index & $\mathrm{meq} \mathrm{O}_{2} / \mathrm{kg}$ \\
$\mathrm{K}_{270}$ spectrophotometry & - \\
$\mathrm{K}_{232}$ spectrophotometry & - \\
$\Delta \mathrm{Kc} \mathrm{spectrophotometry}$ & - \\
Total tocopherols & $\mathrm{mg} / \mathrm{kg}$ \\
$\alpha$-Tocopherol & $\mathrm{mg} / \mathrm{kg}$ \\
$\beta$-Tocopherol & $\mathrm{mg} / \mathrm{kg}$ \\
$\gamma$-Tocopherol & $\mathrm{mg} / \mathrm{kg}$ \\
$\delta$-Tocopherol & $\mathrm{mg} / \mathrm{kg}$ \\
\hline
\end{tabular}

Basic statistical parameters such as minimum, maximum, mean and range were computed for the results of these determinations. Radial plots were used to visualize the dispersion of the data after the exposure of the samples to light treatments of different lengths. 


\subsection{Estimation Models}

Partial Least Square Regression (PLSR) was developed in order to generate estimation models for the oil characteristics determined by the accredited laboratory. Leave-one out cross validation was applied. The selection of the number of latent variables was carried out according to the number at which the values of standard error of calibration (SEC) and the standard error of cross-validation (SECV) diverge. The performance of the regression models was evaluated using the coefficient of determination $\left(R^{2}\right)$.

\section{Results and Discussion}

\subsection{Selected Excitation Wavelength}

The excitation spectra computed on the three-dimensional front-face fluorescence spectra for the seven EVOO samples considered is shown in Figure 2. The excitation spectra showed consistent patterns. The position of the local maxima of the spectra was the same independently of the variety and composition of the oil. The variability found in the intensity of these peaks revealed their ability to sense the differences in the content of the compounds giving rise to the signals. Consequently, filters centered on these wavelengths could be useful for estimation of quality parameters related to such compounds.
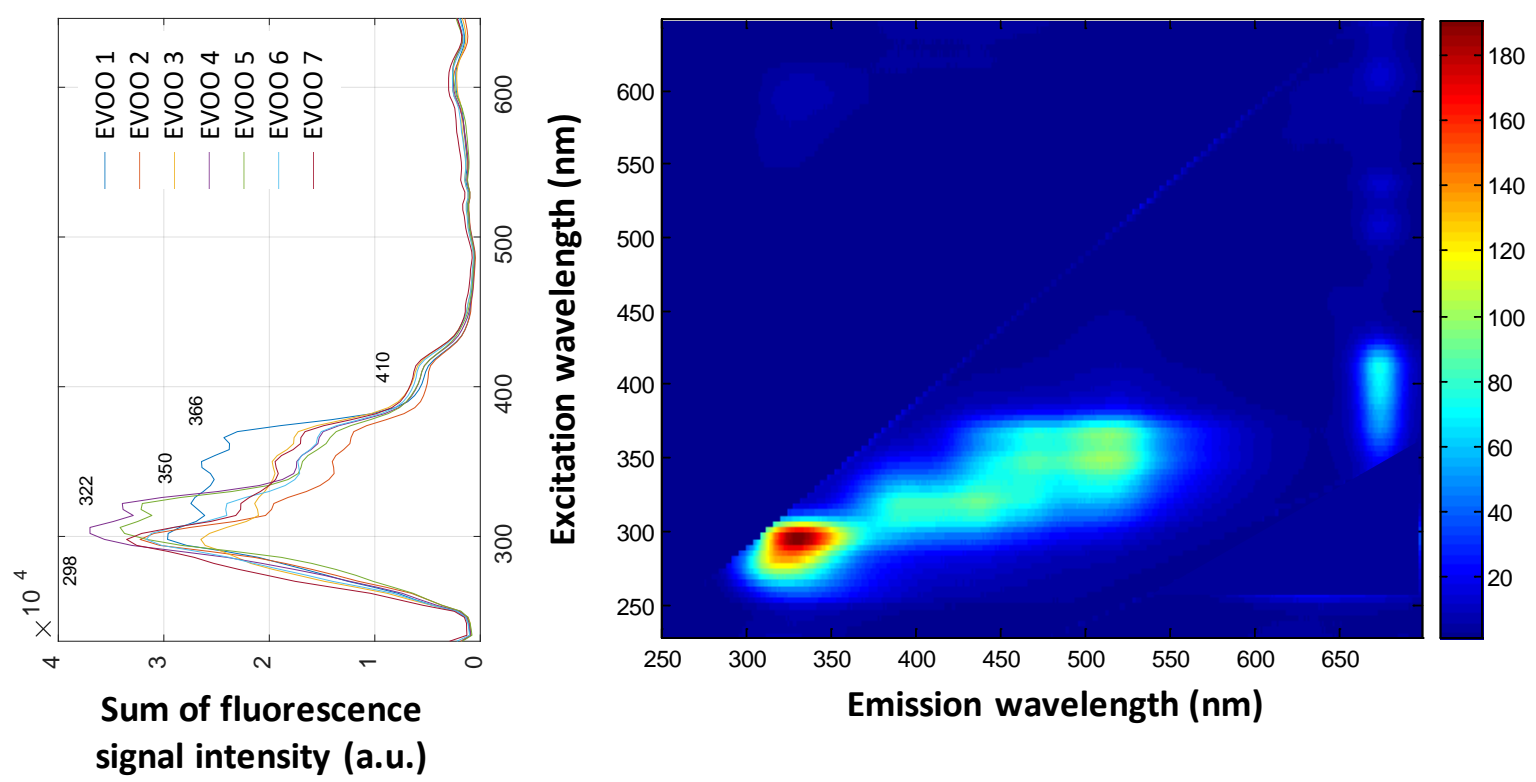

Figure 2. Three-dimensional front-face fluorescence spectroscopy. Left: Excitation spectra of seven extra virgin olive oil (EVOO) samples (wavelengths at local maxima are indicated). Right: example of three-dimensional front-face fluorescence spectra obtained from EVOO 1 sample. Excitation spectra are obtained as the sum of the emission intensity signals along the $x$ axis from 250 to $698.5 \mathrm{~nm}$.

The spectral band that excited any of the fluorophores contained in olive oil ranged from approximately $250 \mathrm{~nm}$ to $450 \mathrm{~nm}$. Local maxima were found at 298, 322, 350, 366 and $410 \mathrm{~nm}$. These findings are in accordance with the literature [16-20]. The band ranging from 270-310 nm excites antioxidant compounds such as tocopherol and polyphenols, which emit light from 300-350 nm. The band ranging from 325-425 nm excites chlorophylls and pheophytins, whose emission band is around $680 \mathrm{~nm}$ [18-21]. A third band of excitation from 274-400 nm corresponds to oxidation products and tocopherols, with a broad emission band from 375-550 $\mathrm{nm}[7,8,18]$.

Finally, in view of the literature and present results three bandpass filters centered at 326, 350 and $365 \mathrm{~nm}$, with a bandwidth (BW) of $10 \mathrm{~nm}$, were selected for further analysis. The objective was to 
develop estimation models for oil quality parameters established in the decision tree illustrated in Table 1.

\subsection{Analysis of the Parameters Obtained in the Laboratory}

Table 5 shows basic statistical features computed from the characteristics determined by the accredited laboratory. Despite the exposure to ambient light for four weeks, the values of acidity, $\mathrm{K}_{270}$ and $\mathrm{K}_{232}$ remained within the limits established for EVOO quality (Table 1). As for the peroxide index, only one sample exceeded the limiting value of $20 \mathrm{meq} \mathrm{O}_{2} / \mathrm{kg}$. This response is attributed to the high levels of antioxidant products, i.e., tocopherols and polyphenols (Table 5), which limited the degradation of the olive oils. In view of the data in Table $5, \alpha$-tocopherol is the predominant form among the total tocopherols.

Table 5. Summary of the results of the chemical analyses of the 24 samples.

\begin{tabular}{|c|c|c|c|c|c|c|c|c|c|}
\hline & $\begin{array}{c}\text { Acidity } \\
\text { (\% ac. } \\
\text { Oleic) }\end{array}$ & $\begin{array}{l}\text { Perox. Index } \\
\left.\text { (meq } \mathrm{O}_{2} / \mathrm{kg}\right)\end{array}$ & $K_{270}$ & $K_{232}$ & $\begin{array}{c}\text { Total } \\
\text { Tocopherols } \\
\text { (mg/kg) }\end{array}$ & $\begin{array}{c}\alpha \\
\text { Tocopherol } \\
(\mathrm{mg} / \mathrm{kg})\end{array}$ & $\begin{array}{c}\beta \\
\text { Tocopherol } \\
(\mathrm{mg} / \mathrm{kg})\end{array}$ & $\begin{array}{c}\gamma \text { Tocopherol } \\
(\mathrm{mg} / \mathrm{kg})\end{array}$ & $\begin{array}{c}\text { Total } \\
\text { Polyphenols } \\
(\mathrm{mg} / \mathrm{kg})\end{array}$ \\
\hline Mean & 0.13 & 9.08 & 0.14 & 1.83 & 244.99 & 233.91 & 2.43 & 8.65 & 563.88 \\
\hline Min. & 0.10 & 3.80 & 0.12 & 1.68 & 165.70 & 157.50 & 1.70 & 6.40 & 410 \\
\hline $\begin{array}{c}\text { Range } \\
\text { (Max.-Min.) }\end{array}$ & 0.05 & 20.20 & 0.04 & 0.48 & 182.50 & 174.60 & 1.60 & 7.10 & 273 \\
\hline
\end{tabular}

Radial diagrams in Figure 3 illustrate the evolution of the peroxide index and the total tocopherols content during light exposure. Each spoke of the polygon corresponds to an initial sample. Peroxide index increased with light exposure, as expected, since the appearance of peroxides is a result of oxidation processes. In contrast, the content of total tocopherols decreased as a consequence of their antioxidant activity.
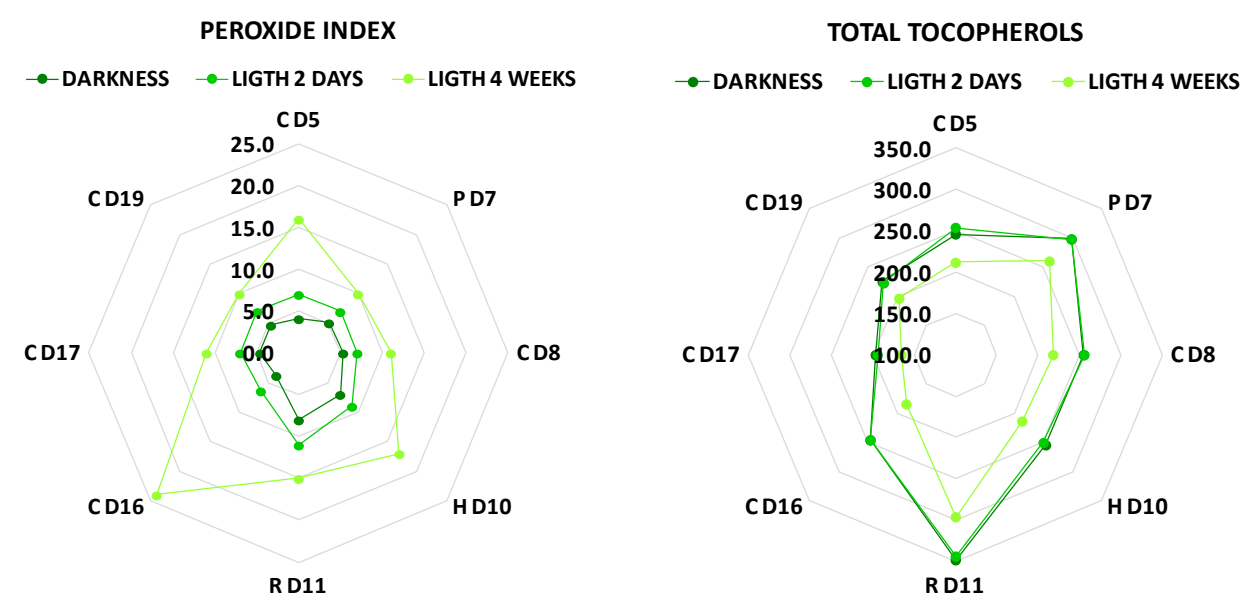

Figure 3. Radial diagrams of peroxide index and total tocopherols content for the eight original samples and the light exposure treatments.

Each sample showed different evolution pattern, which highlighted the importance of the original composition in the strength of the oxidative conditions effect on the olive oil.

\subsection{Analysis of the Fluorescence Spectra}

The spectra obtained at the three different excitation wavelengths consistently showed emission peaks within two differentiated regions (Figure 4). The overall spectral pattern found was similar to that obtained in previous works [10-12] with dominant fluorescence peak at 670 from chlorophyll and pheophytins, together with a broad region between 400-600 nm derived from oxidation products and antioxidants. 


\section{Excitation at $326 \mathrm{~nm}$}

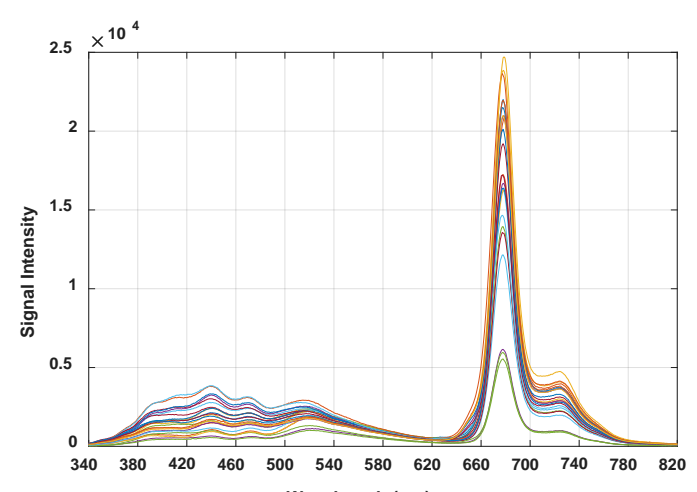

Wavelength $(\mathrm{nm})$

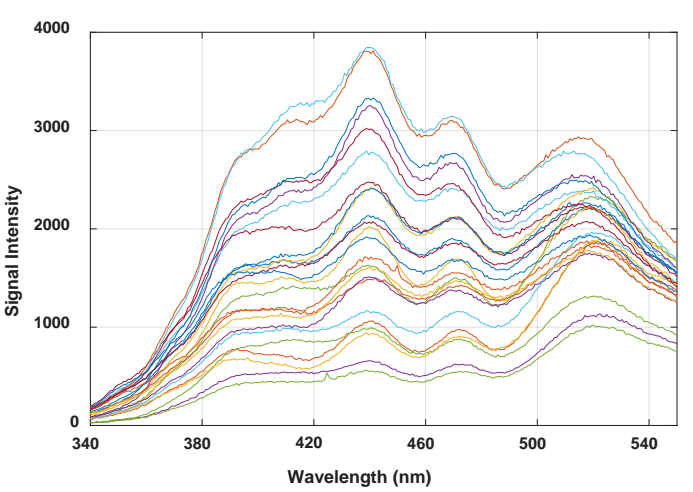

\section{Excitation at $350 \mathrm{~nm}$}
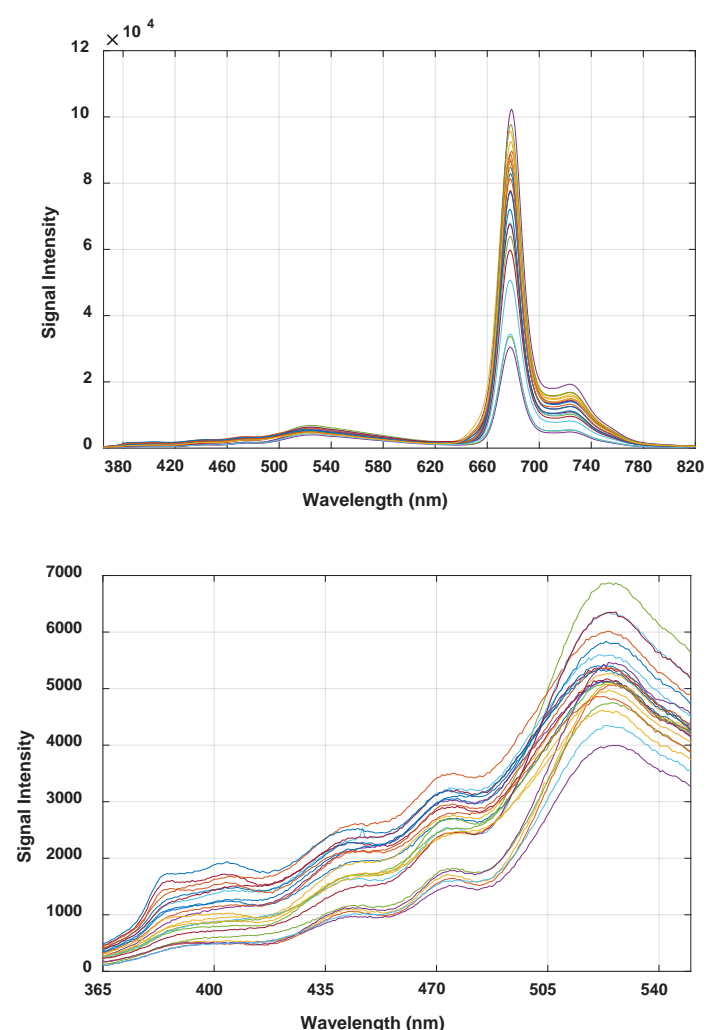

Excitation at $365 \mathrm{~nm}$
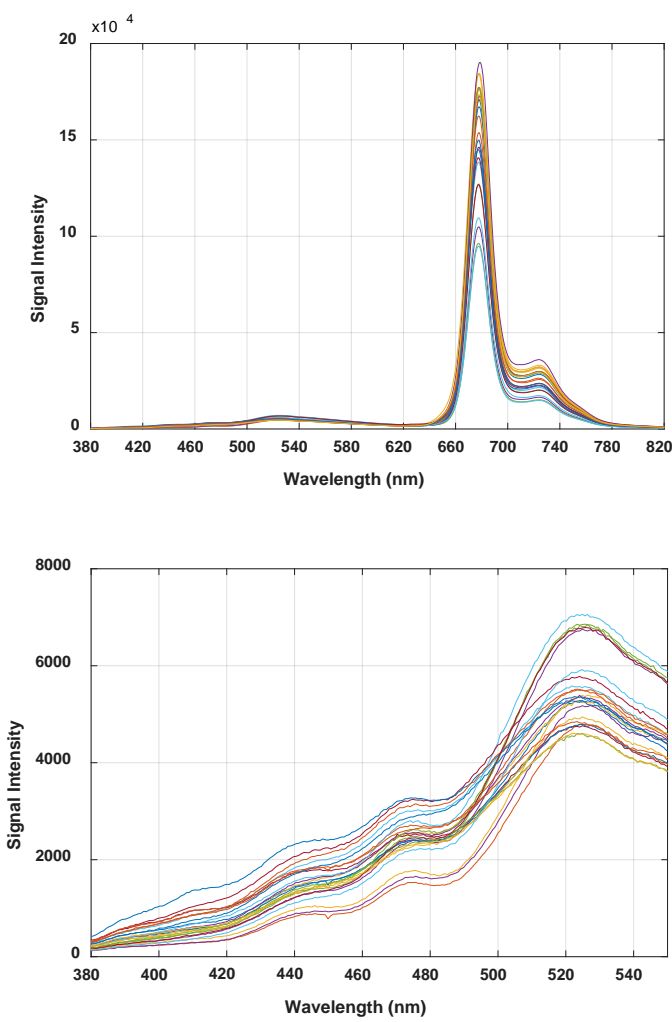

Figure 4. Fluorescence emission spectra. Upper row: fluorescence emission spectra ( $N=24$ samples) obtained at excitation wavelengths centered at 326,350 and 365 $\mathrm{nm}$. Bottom row: detailed emission between $340 \mathrm{~nm}$ and $550 \mathrm{~nm}$. 
In our research the region at shorter wavelengths, which corresponded to oxidation products, showed local maxima located around $385 \mathrm{~nm}, 405 \mathrm{~nm}, 440 \mathrm{~nm}, 470 \mathrm{~nm}$ and $520 \mathrm{~nm}$. According to literature [18-21] the tocopherols also emit around $520 \mathrm{~nm}$. Then, superimposed signals were registered around this wavelength.

Despite these similarities, the intensity of the emission signals showed differences between excitation conditions. Differences were also found in the dominant peaks within the region of the oxidation products and tocopherols (Figure 4 bottom row).

Excitation at $365 \mathrm{~nm}$ achieved the highest emission signals from the chlorophylls. The intensity peak around $520 \mathrm{~nm}$ was clearly dominant within the region of oxidation products, whereas peaks at wavelengths lower than $420 \mathrm{~nm}$ were very subtle. In contrast, when oil samples were excited at $350 \mathrm{~nm}$ the emission spectra showed evident peaks at $385 \mathrm{~nm}$ and $405 \mathrm{~nm}$. Under these excitation conditions, a peak at $520 \mathrm{~nm}$ was dominant as well. Excitation at $326 \mathrm{~nm}$ showed the lowest effectiveness in exciting the fluorescent compounds since the emission intensity was lower along the whole spectra. However, the pattern within the region of oxidation products was noticeably different as peaks at wavelengths shorter than $520 \mathrm{~nm}$ were more preponderant than peaks at $520 \mathrm{~nm}$. These results highlighted that the effectiveness in producing fluorescence signal from the different compounds greatly depended on the selected light of excitation, which is in accordance with the basics of fluorescence spectroscopy [22].

Oxidation products comprise a great variety of compounds. Conjugated and non-conjugated hydroperoxides are products from primary oxidation processes. Secondary oxidation products include low-molecular-weight aldehydes, ketones, alcohols and short-chains hydrocarbons. Therefore, the observed complex fluorescence emission pattern was expected, where signals were produced by a diversity of compounds and were acquired at different wavelengths. Such emissions potentially contained useful information on composition and oxidation status.

For further analysis the emission region below $550 \mathrm{~nm}$ was selected in order to develop multivariate models for the estimation of physico-chemical characteristics related to oxidation status and for the estimation of tocopherols content.

\subsection{Models for Estimating Physico-Chemical Parameters}

Models for the estimation of physico-chemical parameters and tocopherols content were developed for each of the filters considered. The predictive capacity of the models was evaluated through the coefficient of determination $R^{2}$. The results obtained from the PLSR models for each parameter are summarized in Table 6 . The number of latent variables ranged from 2 to 5 . The highest $R^{2}$ values were found for excitation at $350 \mathrm{~nm}$, reaching almost 0.9 in most of the parameters (acidity, peroxide value, $K_{232}$, total tocopherols and isomers). Figures 5 and 6 illustrate detailed results for $K_{232}$ and total tocopherols, both with $R^{2}$ values of 0.88 .

The high $R^{2}$ values achieved was a noticeable result since the estimation models were developed with samples that showed very low degradation and very low variability. Such characteristics involved low emission signals as well as low dispersion of the signals, which may be a hindrance in the development of chemometric models [23]. This performance highlighted the high capability of the fluorescence for detailed characterization of oil samples, in terms of physico-chemical characteristics and antioxidants content.

Other authors found successful results for some physico-chemical characteristics [8]. However, the set of samples included olive oils that showed an important degradation due to heat effects.

Figure 5a shows on the $x$ axis the values of $K_{232}$ determined by the accredited laboratory, versus the values estimated with the partial least squares regression (PLSR) model on the y axis. The scattered data illustrated the importance of the initial composition of the sample in the evolution of the sample characteristics when exposed to oxidation conditions. Some samples exposed to ambient light for four weeks (blue circles) showed lower $K_{232}$ values than others maintained under darkness conditions (red circles). 
Two samples that were correctly estimated (close to the diagonal in the scatterplot) were selected in order to compare their spectra. Figure $5 \mathrm{~b}$ presents the raw spectra of the sample $4 \mathrm{H}$ (red circle) and the sample $5 \mathrm{R}$ (green circle) with low and high $\mathrm{K}_{232}$ respectively. The latter presented higher fluorescence emission over the whole wavelengths range, as it can be expected from its probably higher oxidation status.

\section{Excitation at $350 \mathrm{~nm}$ \\ K232 estimation}

(a)

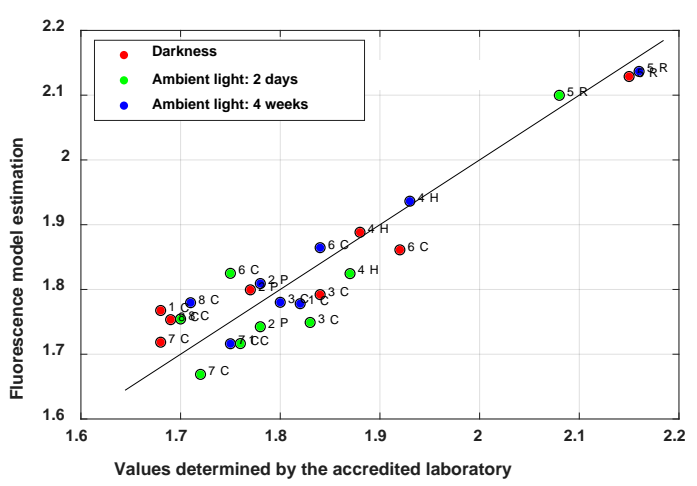

(b)

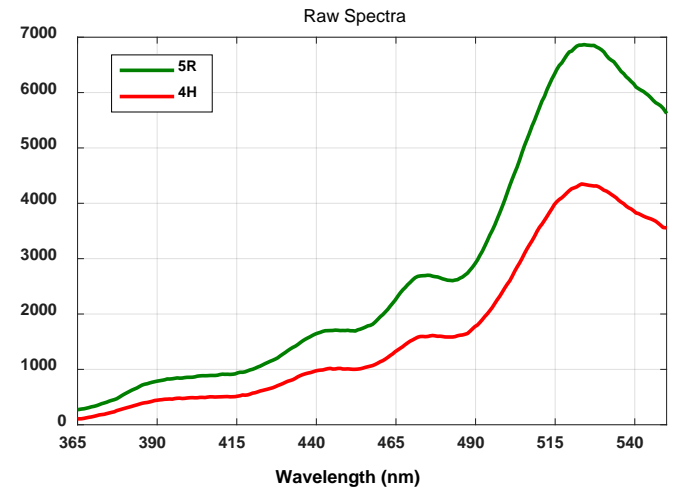

(c)

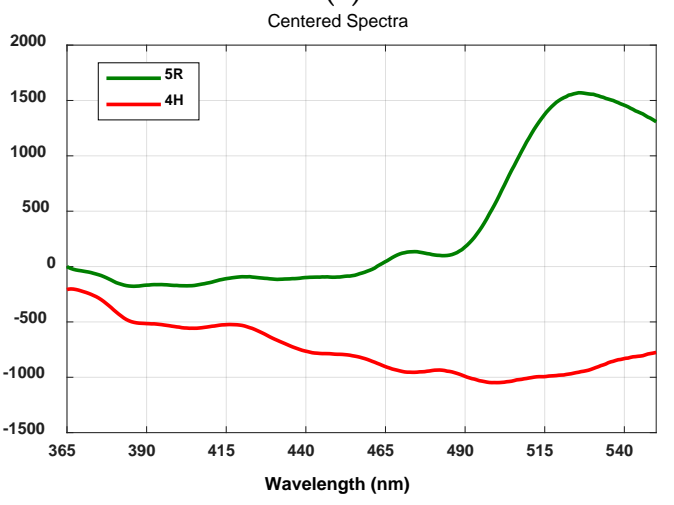

(d)

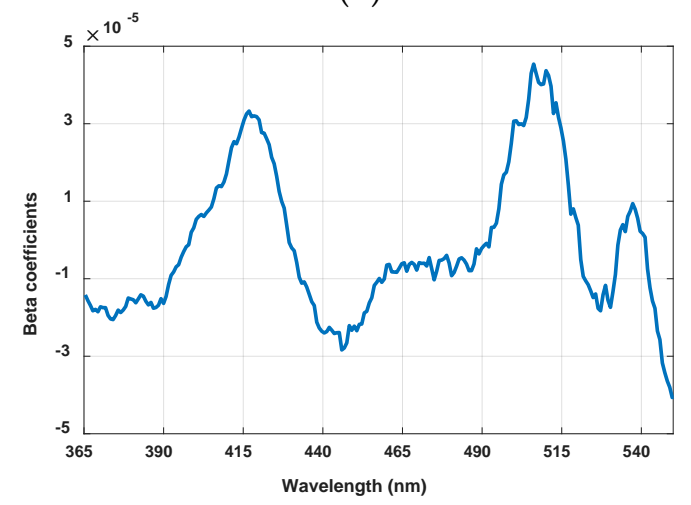

Figure 5. (PLSR) with 4 latent variables for estimation of $K_{232}$ with excitation at $350 \mathrm{~nm}$. (a) $K_{232}$ values determined by the accredited laboratory versus the values estimated with the fluorescence models (colors refer to the three illumination conditions); (b) spectra of 5R (green circle) and $4 \mathrm{H}$ (red circle) of high and low values of $K_{232}$ respectively; (c) the two spectra centered with respect to the average of the whole sampling group $(N=24)$; (d) beta coefficients of the estimation model.

Table 6. Result for partial least squares regression (PLSR) models for each physical-chemical parameter.

\begin{tabular}{|c|c|c|c|c|c|c|}
\hline & $\begin{array}{c}\lambda_{\text {exc }} \\
326 \mathrm{~nm}\end{array}$ & & $\begin{array}{c}\lambda_{\text {exc }} \\
350 \mathrm{~nm}\end{array}$ & & $\begin{array}{c}\lambda_{\text {exc }} \\
365 \mathrm{~nm}\end{array}$ & \\
\hline $\begin{array}{c}\text { Physico-Chemical } \\
\text { Parameter }\end{array}$ & $R^{2}$ & $\begin{array}{c}\text { Latent } \\
\text { Variables }\end{array}$ & $R^{2}$ & $\begin{array}{c}\text { Latent } \\
\text { Variables }\end{array}$ & $R^{2}$ & $\begin{array}{c}\text { Latent } \\
\text { Variables }\end{array}$ \\
\hline Acidity & 0.54 & 3 & 0.84 & 4 & 0.52 & 2 \\
\hline Peroxide index & 0.64 & 3 & 0.67 & 4 & 0.46 & 3 \\
\hline $\mathrm{K}_{232}$ & 0.55 & 5 & 0.88 & 4 & 0.72 & 2 \\
\hline $\mathrm{K}_{270}$ & 0.53 & 5 & 0.30 & 4 & 0.15 & 3 \\
\hline Total tocopherols & 0.43 & 4 & 0.88 & 4 & 0.61 & 2 \\
\hline$\alpha$-Tocopherol & 0.44 & 4 & 0.88 & 4 & 0.61 & 2 \\
\hline$\beta$-Tocopherol & 0.38 & 3 & 0.82 & 2 & 0.84 & 2 \\
\hline$\gamma$-Tocopherol & 0.35 & 5 & 0.86 & 2 & 0.46 & 2 \\
\hline
\end{tabular}


Excitation at $350 \mathrm{~nm}$

Total tocopherols content estimation

(a)

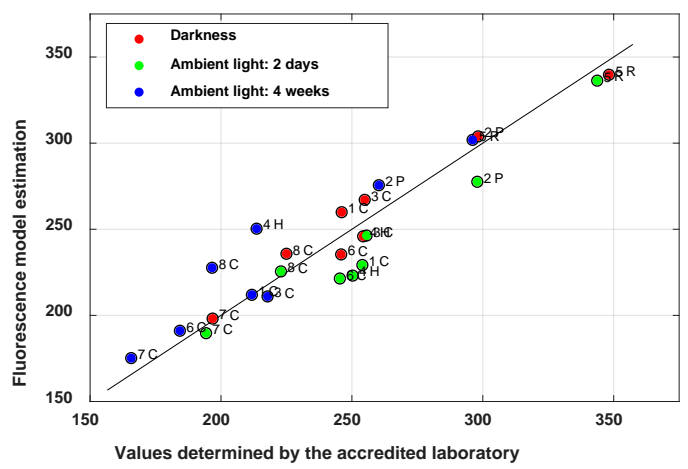

(b)

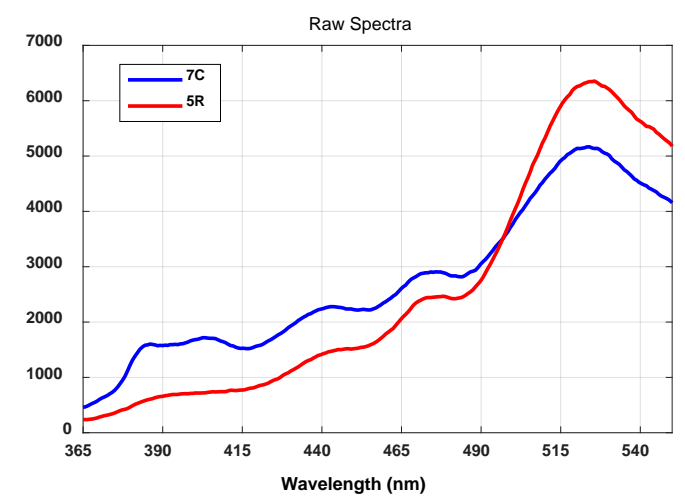

(c)

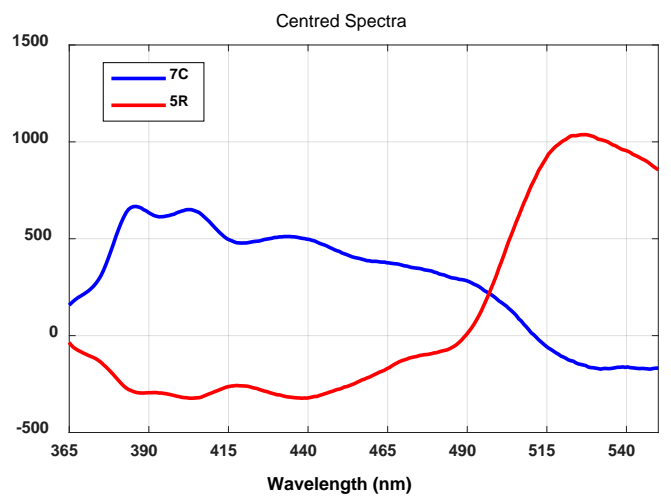

(d)

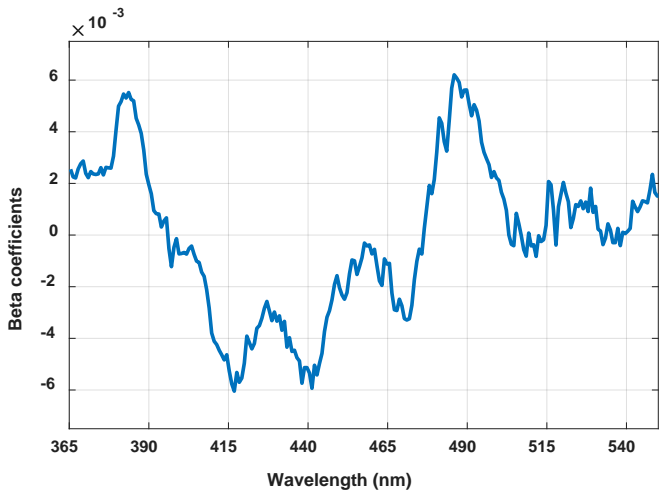

Figure 6. PLSR with 4 latent variables for estimation of total tocopherols content with excitation at 350 $\mathrm{nm}$. (a) Total tocopherols values determined by the accredited laboratory versus the values estimated with the fluorescence models (colors refer to the three illumination conditions); (b) Spectra of 5R (red circle) and 7C (red circle) of high and low total tocopherols content respectively; (c) the two spectra centered with respect to the average of the whole sampling group $(N=24)$; $(\mathbf{d})$ beta coefficients of the estimation model.

PLSR estimation models were computed by using the spectra centered with respect to the average of the whole set of samples $(N=24)$. Figure $5 \mathrm{c}$ shows the corresponding centered spectra of $4 \mathrm{H}$ and $5 \mathrm{R}$ samples. $5 \mathrm{R}$ centered spectrum presented high and positive values between $500 \mathrm{~nm}$ and $560 \mathrm{~nm}$, with a high and positive slope between $500 \mathrm{~nm}$ and $520 \mathrm{~nm}$, whereas $4 \mathrm{H}$ centred spectrum presented negative values along the whole range. Figure $5 \mathrm{~d}$ shows the beta coefficients of the model with relevant wavelengths bands around $420 \mathrm{~nm}$ and around $510-520 \mathrm{~nm}$. The last one is coincident with the positive slope found between $510 \mathrm{~nm}$ and $520 \mathrm{~nm}$ in $5 \mathrm{R}$ centered spectrum.

The beta coefficients of the PLSR model are shown in Figure 5d. For each centered spectrum, the sum of the product of the values at each wavelength multiplied by the corresponding beta coefficient at such wavelength provided the estimation of its $\mathrm{K}_{232}$ (Figure 5a). This sum of products generated high value of estimation of $K_{232}$ for the $5 R$ sample, since within $500 \mathrm{~nm}$ and $520 \mathrm{~nm}$ ranges both spectrum and coefficients were positive. Moreover, beta coefficients computed an approximation to the slope between $500 \mathrm{~nm}$ and $520 \mathrm{~nm}$, which was positive and high for $5 \mathrm{R}$ centered spectrum. On the other hand, the model applied onto the centered spectrum of $4 \mathrm{H}$ produced negative values as beta coefficients were positive and the values of the mean centered spectra were negative, which contributed to its low $\mathrm{K}_{232}$ estimated value. 
Figure 6 illustrates the results for total tocopherols content. Figure 6a shows sample 7C (blue circle, one month storage under indirect illumination) and $5 \mathrm{R}$ (red circle, darkness storage); both were selected for further description as their predicted and observed values were almost coincident. Their tocopherol values were low and high respectively. Figure $6 b, c$ shows the two raw spectra and the same spectra but centered with respect to the average of the 24 samples. The $5 \mathrm{R}$ raw spectrum presented a very high and dominant peak of fluorescence around $520 \mathrm{~nm}$; within the range from 360 to $500 \mathrm{~nm}$ its level of fluorescence was lower than for 7C spectrum. The preponderant emission at $520 \mathrm{~nm}$ of $5 \mathrm{R}$ spectrum could be derived from its higher level of tocopherol. The lower emission at 360 to $500 \mathrm{~nm}$ could be ascribed to lower oxidation status since such sample was stored under darkness conditions.

Figure $6 \mathrm{~d}$ shows the beta coefficients of the model with very well-defined predominant regions and peaks (positive at $380 \mathrm{~nm}$ and between $480 \mathrm{~nm}$ and $500 \mathrm{~nm}$; negative at $420 \mathrm{~nm}$ and $440 \mathrm{~nm}$ approximately). The sum of the products of the spectrum $5 R$ (centered), wavelength by wavelength, by the beta coefficients between $480 \mathrm{~nm}$ and $500 \mathrm{~nm}$ produced high value on the estimation of tocopherol due to the high slope observed in the spectrum at this region. In addition, the negative and high beta coefficients at $420 \mathrm{~nm}$ and $440 \mathrm{~nm}$ multiplied by the negative values of the 5R spectrum at $420 \mathrm{~nm}$ and $440 \mathrm{~nm}$ also produce a high level of tocopherol estimation. The opposite occurred to 7C spectra, where the sum of the products in the range between $480 \mathrm{~nm}$ and $500 \mathrm{~nm}$ contributed to the low tocopherol estimation (because the sum of the products produces negative values) and in the peaks $420 \mathrm{~nm}$ and $440 \mathrm{~nm}$, also produced negative values.

Although further analysis has to be done with a higher number of samples, including higher variability in physico-chemical characteristics and antioxidants content, these are encouraging results since the fluorescence spectroscopy, when a proper wavelength is applied for excitation, has shown great potential to estimate them. At higher oxidation state the signals from the derived products will become more evident, which might involve even better results. As for the estimation of the total tocopherols, the promising results obtained in this study need to be evaluated with samples showing less content.

Most fluorescence research is focused on sensing differences between samples that have undergone drastic conditions of oxidation, or between samples showing significant dissimilarities (as reviewed in the Introduction). Frequently these studies were conducted to identify blended samples prepared in laboratory containing pure EVOO samples and increasing proportions of cheaper oils such as sunflower, seed, or expired olive oils. In contrast, the present research is focused on the quantitative estimation of characteristics and compounds showing slight variation between the samples comprising the analyzed set. Moreover, the present results could be implemented to identify adulterations as well.

In addition, this procedure could be flexible and adaptable to the requirements of the different agents within the value chain of olive oil (producers, distributors, exporters, importers, etc.). Such a method could allow a pre-characterization of samples prior to the application of the battery of regulated analytical determinations. This could facilitate decision-making in the handling of samples and promote the reduction of analytical procedures, which are slow and involve management of chemical residues, with the corresponding reduction of costs.

\section{Conclusions}

This work revealed the potential of fluorescence spectroscopy to estimate physico-chemical characteristics related to the oxidation state of EVOO and antioxidant content such as tocopherols. Internal changes produced in the composition of olive oil as a result of exposure to light are manifested in changes in the shape of the fluorescence emission spectra within the range from $365 \mathrm{~nm}$ to $550 \mathrm{~nm}$.

Despite the minimum oxidation of the samples and the subtle differences between samples, the specificity and sensibility of fluorescence spectroscopy provided useful complex emission patterns. Signals were produced by a diversity of compounds and each specific compound presented an optimal range of excitation wavelengths. The computation of excitation spectra revealed local maxima that 
allowed identifying the wavelengths bands that were more effective in producing signals with relevant information. Excitation at $350 \mathrm{~nm}$ provided the most useful emission spectra since the estimation models provided $R^{2}$ close to 0.9 for acidity, $\mathrm{K}_{232}$ and total tocopherol content. The $\mathrm{R}^{2}$ was close to 0.7 for the peroxide index.

Further analysis is needed with a higher number of samples, including more varieties, more varied composition and oxidation status in order to validate and to improve the estimation models. This work constitutes the first step for developing a future analytical tool, easy to implement, fast, with a low economic and environmental cost, based on fluorescence spectroscopy.

Author Contributions: Conceptualization, N.H.-S., B.D. and L.L.; Data curation, P.B., N.H.-S. and L.L.; Formal analysis, P.B.; N.H.-S. and L.L.; Funding acquisition, B.D.; Investigation, P.B., N.H.-S. and L.L.; Methodology, P.B., N.H.-S., B.D. and L.L.; Project administration, B.D.; Resources, N.H.-S.; Software, N.H.-S., B.D.; Supervision, N.H.-S., B.D. and L.L.; Writing-original draft, P.B., N.H.-S. and L.L.; Writing-review \& editing, N.H.-S. and L.L. All authors have read and agreed to the published version of the manuscript.

Funding: This research received no external funding.

Acknowledgments: Authors gratefully acknowledge David Perez of the mill LA PONTEZUELA SLU (Spain) for providing the EVOO samples; and MULTISCAN TECHNOLOGIES SL (Spain) for technical support. We also gratefully acknowledge Jean-Michel Roger from IRSTEA (Montpellier, France) for his support in the spectral analyses and advice regarding the experimental set-up.

Conflicts of Interest: The authors declare no conflict of interest.

\section{References}

1. International Olive Council. Available online: www.internationaloliveoil.org (accessed on 15 April 2019).

2. EC, European Community Commission. Regulation no. 2568/1991 on the characteristics of olive and olive pomace oils and their analytical methods. Off. J. Eur. Commun. 1991, L248, 1-83.

3. International Olive Council. Trade Standard Applying to Olive Oils and Olive Pomace Oils. COI/T.15/NC No 3/Rev. 12. 2018. Available online: https://www.internationaloliveoil.org/what-we-do/chemistrystandardisation-unit/standards-and-methods (accessed on 15 April 2019).

4. Karoui, R.; Blecker, C. Fluorescence spectroscopy measurement for quality assessment of food systems a review. Food Bioprocess Technol. 2011, 4, 364-386. [CrossRef]

5. Meenu, M.; Cai, Q.; Xu, B. A critical review on analytical techniques to detect adulteration of extra virgin olive oil. Trends Food Sci. Technol. 2019, 91, 391-408. [CrossRef]

6. Gorzynik-Debicka, M.; Przychodzen, P.; Cappello, F.; Kuban-Jankowska, A.; Marino Gammazza, A.; Knap, N.; Wozniak, M.; Gorska-Ponikowska, M. Potential Health Benefits of Olive Oil and Plant Polyphenols. Int. J. Mol. Sci. 2018, 19, 686. [CrossRef] [PubMed]

7. Sikorska, E.; Khmelinskii, I.; Sikorski, M. Analysis of Olive Oils by Fluorescence Spectroscopy: Methods and Applications, Olive Oil-Constituents, Quality, Health Properties and Bioconversions; Boskou, D., Ed.; IntechOpen: Rijeka, Croatia, 2012. [CrossRef]

8. Guzmán, E.; Baeten, V.; Pierna, J.A.F.; García-Mesa, J.A. Evaluation of the overall quality of olive oil using fluorescence spectroscopy. Food Chem. 2015, 173, 927-934. [CrossRef] [PubMed]

9. Lleó, L.; Hernández-Sánchez, N.; Ammari, F.; Roger, J.M. 3D front-face fluorescence spectroscopy for characterization of extra virgin olive oil and olive oil according to the spectral pattern. CIGR J. 2016, 18, 190-199.

10. Hernández-Sánchez, N.; Lleó, L.; Ammari, F.; Cuadrado, T.R.; Roger, J.M. Fast Fluorescence Spectroscopy Methodology to Monitor the Evolution of Extra Virgin Olive Oils under Illumination. Food Bioprocess Technol. 2017, 10, 949-961. [CrossRef]

11. Mishra, P.; Lleó, L.; Cuadrado, T.; Ruiz-Altisent, M.; Hernández-Sánchez, N. Monitoring oxidation changes in commercial extra virgin olive oils with fluorescence spectroscopy-based prototype. Eur. Food Res. Technol. 2018, 244-565. [CrossRef]

12. Ali, H.; Saleem, M.; Answer, M.R.; Khan, S.; Ullah, R.; Bilal, M. Validation of Fluorescence Spectroscopy to Detect Adulteration of Edible Oil in Extra Virgin Olive Oil (EVOO) by Applying Chemometrics. Appl. Spectrosc. 2018, 72, 1371-1379. [CrossRef] [PubMed] 
13. Lastra-Mejias, M.; Izquierdo, M.; Torreblanca-Zanca, A.; Aroca-Santosa, R.; Cancilla, J.C.; Sepulveda-Diaz, J.E.; Torrecilla, J.S. Cognitive fluorescence sensing to monitor the storage conditions and locate adulterations of extra virgin olive oil. Food Control 2010, 103, 48-58. [CrossRef]

14. Dankowska, A.; Kowalewski, W. Comparison of different classification methods for analyzing fluorescence spectra to characterize type and freshness of olive oils. Eur. Food Res. Technol. 2019, 245, 745-752. [CrossRef]

15. Díaz, G.; Pega, J.; Primrose, D.; Sancho, A.M.; Nanni, M. Effect of Light Exposure on Functional Compounds of Monovarietal Extra Virgin Olive Oils and Oil Mixes During Early Storage as Evaluated by Fluorescence Spectra. Food Anal. Methods 2019. [CrossRef]

16. Ammari, F. Etude et Amélioration de la Stabilité des Huiles Végétale Cours du Chauffage Apport des Méthodes Chimiométriques dans 1'Analyse des Données. Ph.D. Thesis, AgroParisTech, Universite Paris-Saclay, Paris, France, 2012.

17. Ammari, F.; Rimbaud-Bouveresse, J.; Boughanmi, D.; Rutledge, D.N. Study of the heat stability of sunflower oil enriched in natural antioxidants by different analytical techniques and front-face fluorescence spectroscopy combined with Independent Components Analysis. Talanta 2012, 99, 323-329. [CrossRef] [PubMed]

18. Kyriakidis, N.B.; Skarkalis, P. Fluorescence spectra measurement of olive oil and other vegetable oils. J. AOAC Int. 2000, 83, 1435-1439. [PubMed]

19. Sikorska, E.; Khmelinskii, I.V.; Sikorski, M.; Caponio, F.; Bilancia, M.T.; Pasqualone, A.; Gomes, T. Fluorescence spectroscopy in monitoring of extra virgin olive oil during storage. International. J. Food Sci. Technol. 2008, 43, 52-61. [CrossRef]

20. Zandomeneghi, M.; Carbonaro, L.; Caffarata, C. Fluorescence of vegetable oils: Olive oils. J. Agric. Food Chem. 2005, 53, 759-766. [CrossRef] [PubMed]

21. Sikorska, E.; Górecki, T.; Khmelinskii, I.V.; Sikorski, M.; Koziol, J. Classification of edible oils using synchronous scanning fluorescence spectroscopy. Food Chem. 2005, 89, 217-225. [CrossRef]

22. Kumke, M.; Löhmannsröben, H.G.; Buschmann, C.; Langsdorf, G.; Lichtenthaler, H.K.; Saito, Y.; Rizkallaf, J.; Lakhal, L.; Birlouez-Aragon, I.; Kameoka, T.; et al. Fluorescence. In Optical Monitoring of Fresh and Processed Agricultural Crops, 1st ed.; Zude, M., Ed.; CRC Press, Taylor and Francis Group: Boca Raton, FL, USA, 2008; pp. 251-376.

23. Zeaiter, M.; Roger, J.M.; Bellon-Maurel, V.; Rutledge, D.N. Robustness of models developed by multivariate calibration. Part I: The assessment of robustness. TrAC Trends Anal. Chem. 2004, 23, 157-170. [CrossRef] 